\title{
A REMARK ON THE GENUS OF CURVES IN $\mathbf{P}^{4}$
}

\author{
VINCENZO DI GENNARO
}

\begin{abstract}
Let $C$ be an irreducible, reduced, non-degenerate curve, of arithmetic genus $g$ and degree $d$, in the projective space $\mathbf{P}^{4}$ over the complex field. Assume that $C$ satisfies the following flag condition of type $(s, t): C$ does not lie on any surface of degree $<s$, and on any hypersurface of degree $<t$. Improving previous results, in the present paper we exhibit a CastelnuovoHalphen type bound for $g$, under the assumption $s \leq t^{2}-t$ and $d \gg t$. In the range $t^{2}-2 t+3 \leq s \leq t^{2}-t, d \gg t$, we are able to give some information on the extremal curves. They are arithmetically Cohen-Macaulay curves, and lie on a flag like $S \subset F$, where $S$ is a surface of degree $s, F$ a hypersurface of degree $t, S$ is unique, and its general hyperplane section is a space extremal curve, not contained in any surface of degree $<t$. In the case $d \equiv 0$ (modulo $s$ ), they are exactly the complete intersections of a surface $S$ as above, with a hypersurface. As a consequence of previous results, we get a bound for the speciality index of a curve satisfying a flag condition.
\end{abstract}

Keywords and phrases: Genus of a complex projective curve, CastelnuovoHalphen Theory, Flag condition.

MSC2010: Primary 14N15; 14N05. Secondary 14H99.

\section{INTRODUCTION}

Let $C$ be an irreducible, reduced, non-degenerate curve, of arithmetic genus $g$ and degree $d$, in the projective space $\mathbf{P}^{4}$ over the complex field. Assume that $C$ satisfies the following flag condition of type $(s, t)$ : $C$ does not lie on any surface of degree $<s$, and on any hypersurface of degree $<t$. Under the assumption $s>t^{2}-t$ and $d>\max \left(12(s+1)^{2}, s^{3}\right)$, in [2, Theorem], one proves a sharp upper bound $G(d, s, t)$ for $g$ (for the definition of $G(d, s, t)$, see Section 2, (ii), below). In the present paper, we prove that this bound $G(d, s, t)$ applies also when $s \leq t^{2}-t$ and $d \gg t$. More precisely, we prove the following:

Theorem 1.1. Let $C$ be an irreducible, reduced, non-degenerate curve, of arithmetic genus $g$ and degree $d$, in the projective space $\mathbf{P}^{4}$ over the complex field. Assume $C$ is not contained in any hypersurface of degree $<t(t \geq 3)$, and in any surface of degree $<s(s \geq 3)$. Define $\alpha, \beta, m$ and $\epsilon$ by dividing $s-1=\alpha t+\beta$, $0 \leq \beta<t$, and $d-1=m s+\epsilon, 0 \leq \epsilon<s$. Assume $s \leq t^{2}-t$ and $d>d_{0}$, where

$$
d_{0}:=\left\{\begin{array}{l}
32 t^{4} \quad \text { if } s \leq 2 t-3 \\
8 s t^{4} \quad \text { if } s \geq 2 t-3 \text { and } \beta<t-\alpha-2, \\
\max \left(12(s+1)^{2}, s^{3}\right) \quad \text { if } s \geq 2 t-3 \text { and } \beta \geq t-\alpha-2 .
\end{array}\right.
$$

One has: 
- if either $s \leq 2 t-3$ or $s \geq 2 t-3$ and $\beta<t-\alpha-2$ or $s \geq 2 t-3$ and $\beta>t-\alpha-2$ and either $s-\epsilon-1<\alpha+\beta+2-t$ or $\beta(\alpha+\beta+2-t) \leq s-\epsilon-1<(\beta+1)(\alpha+\beta+2-t)$, then $g<G(d, s, t)$;

- otherwise $g<G(d, s, t)+4 t^{3}$.

The proof relies on the quoted result [2, Theorem], combined with a purely arithmetic argument, inspired by a remark by Ellinsgrud and Peskine [8, p. 2, (B)].

Unfortunately, we are not able to determine the sharp bound. However, in the range $t^{2}-2 t+3 \leq s \leq t^{2}-t$ (and $d \gg s$ ), we are able to give some information on the curves verifying a flag condition, with maximal genus, and to determine the sharp bound in the particular case $d \equiv 0$ (modulo $s$ ). As in the case $s>t^{2}-t$, a hierarchical structure of the family of curves with maximal genus, verifying flag conditions, emerges ([2], 1], 4]). In fact, we prove the following result (the number $\beta$ is defined in the claim of of Theorem 1.1).

Theorem 1.2. Let $C$ be an irreducible, reduced, non-degenerate curve, of arithmetic genus $g$ and degree $d$, in the projective space $\mathbf{P}^{4}$ over the complex field. Assume $C$ is not contained in any hypersurface of degree $<t(t \geq 3)$, and in any surface of degree $<s(s \geq 3)$. Assume $t^{2}-2 t+3 \leq s \leq t^{2}-t, d>s^{4}$, and that $g$ is maximal with respect previous flag condition. Then one has:

$$
g=G(d, s, t)-\frac{d}{s}(\beta-1)+O(1),
$$

where $|O(1)| \leq 2 t^{3}+s^{3}$. Moreover, $C$ is arithmetically Cohen-Macaulay, and lies on a flag $S \subset F$, where $S$ is a surface of degree $s, F$ a hypersurface of degree $t, S$ is unique, and its general hyperplane section is a space extremal curve not contained in any surface of degree $<t$. In the case $\epsilon=s-1$ and $d=(m+1) s$, one has

$$
g=G(d, s, t)-\frac{d}{s}(\beta-1),
$$

and $C$ is the complete intersection of $S$, with a hypersurface of degree $m+1$.

The proof follows combining various results in Castelnuovo-Halphen Theory ( 9 , [10, [7, 2], 4]). In particular, it relies on the fact that the theory of space curves, of degree $s$ and not contained in any surface of degree $<t$, in the range $t^{2}-2 t+3 \leq$ $s \leq t^{2}-t$, is quite similar to the theory in the classic range $s>t^{2}-t([9],[10,[7])$. Moreover, in order to study the sharp case $d \equiv 0$ (modulo $s$ ), we use the same argument as in the proof of [2, Proposition 15, p. 130], taking into account that it works well also for $s \geq t^{2}-2 t+3$. We have in mind to apply this analysis also for the remaining cases $0 \leq \epsilon<s-1$, in a forthcoming paper.

As a consequence of previous results, we get the following bound for the speciality index. Recall that, for a projective integral curve $C \subset \mathbf{P}^{N}$, one defines the speciality index $e(C)$ of $C$ as the maximal integer $e$ such that $h^{0}\left(C, \omega_{C}(-e)\right)>0$, where $\omega_{C}$ denotes the dualizing sheaf of $C$. The number $d_{0}$, appearing in the claim, is defined in (11).

Corollary 1.3. Let $C$ be an irreducible, reduced, non-degenerate curve, of degree $d$, in the projective space $\mathbf{P}^{4}$ over the complex field. Assume $C$ is not contained in any 
hypersurface of degree $<t(t \geq 3)$, and in any surface of degree $<s(s \geq 3)$. Let $e(C)$ denote the index of speciality of $C$. Assume $s \leq t^{2}-t$ and $d>\max \left(d_{0}, 6 s t^{4}\right)$. Then one has:

$$
e(C) \leq \frac{d}{s}+\frac{s}{t}+t-5
$$

Moreover, if $d \equiv 0$ (modulo $s), s \leq t^{2}-t$, and $d>s^{4}$, then one has:

$$
e(C) \leq \frac{d}{s}+2 t-7 \text {. }
$$

In the range $t^{2}-2 t+3 \leq s \leq t^{2}-t$, the bound (4) is sharp. In fact, every curve, complete intersection of a surface of degree $s$, whose general hyperplane section is a space extremal curve not contained in any surface of degree $<t$, with a hypersurface of degree $m+1$, attains the bound.

Taking into account that $d \cdot e(C) \leq 2 g-2(g:=$ the arithmetic genus of $C)$, previous corollary easily follows from Theorem 1.1] and Theorem[1.2. In the range $s>t^{2}-t$ (and $d>\max \left(\frac{2}{3} s^{4}, 12(s+1)^{2}\right)$ ), the bound (3) is already known, and it is sharp [5. Theorem B, and Remark (i), p. 97]. We do not know whether a curve with maximal speciality given by (4), is necessarily a complete intersection as above.

As for the numerical assumptions appearing in previous results, they are certainly not the sharpest for our purposes. They are only of the simplest form we were able to conceive.

\section{Notations AND PRELIMINARIES For TheOREM 1.1}

In this section we establish some notation, and collect some numerical results (i.e. (6), Lemma 2.1 (8), and Lemma 2.2 below), which we need in the proof of Theorem 1.1. For the proof of these results, which consists in long and elementary calculations, we refer to the Appendix at the end of the paper (Section 6).

(i) Set $\tau:=\alpha+1$. Observe that if $s \leq t^{2}-t$, then $0 \leq \alpha \leq t-2,1 \leq \tau \leq t-1$, and

$$
s>\tau^{2}-\tau .
$$

Define $\alpha^{\prime}$ and $\beta^{\prime}$ by dividing $s-1=\alpha^{\prime} \tau+\beta^{\prime}, 0 \leq \beta^{\prime}<\tau$. Let $x$ be the unique integer $0 \leq x \leq t-1$ such that $t-(x+1)(\alpha+1) \leq \beta<t-x(\alpha+1)$. Then we have $\alpha^{\prime}=t-1-x$, and $\beta^{\prime}=\beta-[t-(x+1)(\alpha+1)]$.

(ii) As in [2, p. 120], we define the numbers:

$$
G(d, s, t):=\frac{d^{2}}{2 s}+\frac{d}{2}\left[\frac{s}{t}+t-5-\frac{(t-1-\beta)(1+\beta)(t-1)}{s t}\right]+\rho+1,
$$

and

$$
G(d, s, \tau):=\frac{d^{2}}{2 s}+\frac{d}{2}\left[\frac{s}{\tau}+\tau-5-\frac{\left(\tau-1-\beta^{\prime}\right)\left(1+\beta^{\prime}\right)(\tau-1)}{s \tau}\right]+\rho^{\prime}+1,
$$

where $\rho=\rho(s, t, \epsilon)$ and $\rho^{\prime}=\rho(s, \tau, \epsilon)$ are "constant terms", for whose definitions we refer to the Appendix, (i). When $s \leq t^{2}-t$, one has

$$
|\rho| \leq 2 t^{3}, \quad\left|\rho^{\prime}\right| \leq 2 t^{3} .
$$


Lemma 2.1. 1) If $t+1 \leq s \leq t^{2}-t$ and $\beta<t-\alpha-2$ and $d>8 s t^{4}$, then $G(d, s, \tau)<G(d, s, t)$.

2) If $s \leq t^{2}-t$ and $\beta>t-\alpha-2$ and either $s-\epsilon-1<\alpha+\beta+2-t$ or $\beta(\alpha+\beta+2-t) \leq s-\epsilon-1<(\beta+1)(\alpha+\beta+2-t)$, then $G(d, s, \tau)=G(d, s, t)$.

3) If either $s \leq t$ or $t+1 \leq s \leq t^{2}-t$ and $t-\alpha-2 \leq \beta$, then $G(d, s, \tau)=$ $G(d, s, t)+\left(\rho^{\prime}-\rho\right) \leq G(d, s, t)+4 t^{3}$.

(iii) We recall also the definition of the bound $G$ for the genus of a curve in $\mathbf{P}^{4}$ of degree $d$, not contained in any surface of degree $<s$ (compare with [1, p. 230-231, and p. 241, Theorem 5.1], and [5. p. 91-92, (4) and $\left.\left(4^{\prime}\right)\right]$ ). Define $w$ and $w_{1}$ by dividing $s-1=2 w+w_{1}, 0 \leq w_{1}<2$. Then we have

$$
G:=\frac{d^{2}}{2 s}+\frac{d}{2}\left(\frac{2 \pi-2}{s}-1\right)+R,
$$

where $\pi=w\left(w-1+w_{1}\right)$, and $R=R(s, \epsilon)$ is a constant term (for the definition, see Appendix, (ii)) such that

$$
|R| \leq s^{2}
$$

Lemma 2.2. If $3 \leq s \leq 2 t-3$ and $d>32 t^{4}$, then $G<G(d, s, t)$.

\section{The Proof of Theorem 1.1}

Proof of Theorem 1.1. First assume $s \leq 2 t-3$. By [1, p. 241, Theorem 5.1], we know that $g \leq G$. Therefore, in this case our claim follows from Lemma 2.2,

Next assume $s \geq 2 t-3$ and $\beta<t-\alpha-2$. In this case we may assume also that $t+1 \leq s$, otherwise $s=t=3$, and we fall back in the previous case. If $t+1 \leq s$, then $\alpha \geq 1$, hence $\tau \geq 2$. Moreover, $\tau<t$. Therefore, $C$ is not contained in any hypersurface of degree $<\tau$. Since $s>\tau^{2}-\tau$ (compare with (5)), we may apply [2, Theorem], and deduce $g \leq G(d, s, \tau)$. By Lemma 2.1 1), we get $g<G(d, s, t)$.

Now assume $s \geq 2 t-3$ and $\beta>t-\alpha-2$ and either $s-\epsilon-1<\alpha+\beta+2-t$ or $\beta(\alpha+\beta+2-t) \leq s-\epsilon-1<(\beta+1)(\alpha+\beta+2-t)$. As before, we have $g \leq G(d, s, \tau)$. In this case, Lemma 2.1, 2), says that $G(d, s, \tau)=G(d, s, t)$. Therefore, we get $g \leq G(d, s, t)$. We cannot have $g=G(d, s, t)$, otherwise, by [2, Theorem], $C$ should be contained in a hypersurface of degree $\tau<t$.

Finally, in the remaining cases, as before we have $g \leq G(d, s, \tau)$. By Lemma 2.1, $3)$, it follows that $g \leq G(d, s, t)+4 t^{3}$. Again, we cannot have $g=G(d, s, t)+4 t^{3}$, otherwise, by [2, Theorem], $C$ should be contained in a hypersurface of degree $\tau<t$. 


\section{Notations And PREliminaries for Theorem 1.2 AND Corollary 1.3}

In order to prove Theorem 1.2 and Corollary 1.3 , in this section we recall some properties of arithmetically Cohen-Macaulay varieties. Moreover, we recall the main results of the theory of space curves of degree $s$, not contained in any surface of degree $<t$, in the range $t^{2}-2 t+3 \leq s \leq t^{2}-t$ (see [9], [10, p. 219], [7, 10.8. Teorema, p. 56]).

(i) If $V \subseteq \mathbf{P}^{N}$ is an integral projective variety, we denote by $h_{V}(t)$ and $p_{V}(t)$ the Hilbert function and the Hilbert polynomial of $V$. We denote by $p_{a}(V)$ its arithmetic genus. The variety $V$ is said to be arithmetically Cohen-Macaulay (shortly a.C.M.) if all the restriction maps $H^{0}\left(\mathbf{P}^{N}, \mathcal{O}_{\mathbf{P}^{N}}(i)\right) \rightarrow H^{0}\left(V, \mathcal{O}_{V}(i)\right)(i \in \mathbf{Z})$ are surjective, and $H^{j}\left(V, \mathcal{O}_{V}(i)\right)=0$ for all $i \in \mathbf{Z}$ and $1 \leq j \leq \operatorname{dim} V-1$ ([11, 9-8], 6, p. 84]). If $\operatorname{dim} V \geq 2$, then $V$ is a.C.M. if and only if its general hyperplane section is. If $V$ is a curve of degree $d$, and $V^{\prime}$ denotes its general hyperplane section, then

$$
p_{a}(V) \leq \sum_{i=1}^{+\infty} d-h_{V^{\prime}}(i)
$$

and equality occurs if and only if $V$ is a.C.M. [6, p. 83-84]. Moreover, using the diagram in [1, p. 232], one may prove that if $V$ is an a.C.M. curve, and $e(V)$ denotes its speciality index, then:

$$
e(V)=\max \left\{n: h_{V^{\prime}}(n)<d\right\}-1 .
$$

(ii) Fix integers $s, t \geq 3$, with $t^{2}-2 t+3 \leq s \leq t^{2}-t$. Let $\Sigma \subset \mathbf{P}^{3}$ be an integral curve, of degree $s$, not contained in any surface of degree $<t$, and of maximal arithmetic genus, that we denote by $P=P(s, t)$ (compare with [10, p. 219], [9, p. 43-49], 7, 10.8. Teorema, p. 56], and (11) below). Set $\nu:=s-\left(t^{2}-2 t+3\right)(=\beta-2)$. $\Sigma$ is an a.C.M. curve, contained in a surface of degree $t$, with caractère numérique $\left(n_{0}, n_{1}, \ldots, n_{t-1}\right)$ given by:

$$
n_{i}:=\left\{\begin{array}{l}
2 t-3-i \quad \text { if } 0 \leq i \leq t-3-\nu, \\
2 t-2-i \quad \text { if } t-2-\nu \leq i \leq t-2, \\
t \quad \text { if } i=t-1
\end{array}\right.
$$

([9, p. 40, p. 45], [7, p. 20, and proof of 10.4: Lemma, p. 53]). If $h=h(n)$ denotes the Hilbert function of the general plane section of $\Sigma$, then one has, for every integer $n$,

$$
h(n)=\sum_{i=0}^{t-1}\left[(n-i+1)_{+}-\left(n-n_{i}+1\right)_{+}\right],
$$

where $(x)_{+}:=\max (0, x)$ [7, 3.7: Lemma, p. 20]. It follows that:

$h(n)=\left(\begin{array}{c}n+2 \\ 2\end{array}\right)-2\left(\begin{array}{c}n+2-t \\ 2\end{array}\right)+\left(\begin{array}{c}n-2 t+4 \\ 2\end{array}\right)-\left(\begin{array}{c}n+1-t \\ 1\end{array}\right)-\left(\begin{array}{c}n+1-t-\nu \\ 1\end{array}\right)$

for every $n$, where we assume $\left(\begin{array}{l}a \\ b\end{array}\right)=0$ if $a<b$. Notice that

$$
h(2 t-5)=s-1, \quad h(2 t-4)=s .
$$


We have:

$$
p_{a}(\Sigma)=P(s, t)=\sum_{i=1}^{+\infty} s-h(i)=t^{3}-5 t^{2}+(\beta+7) t+\frac{\beta^{2}-7 \beta}{2}-1 .
$$

Comparing with (16) (see below) we get:

$$
H(s, t)=P(s, t)+\beta-1 .
$$

Therefore, we may write (compare with (17)):

$$
G(d, s, t)=\frac{d^{2}}{2 s}+\frac{d}{2 s}(2 P-2-s)+\frac{d}{s}(\beta-1)+\rho+1 .
$$

Observe that, if $d=(m+1) s$, then $\rho=0$, and therefore, in this case, we have:

$$
G(d, s, t)=\frac{d^{2}}{2 s}+\frac{d}{2 s}(2 P-2-s)+\frac{d}{s}(\beta-1)+1 .
$$

\section{The Proof of Theorem 1.2 And of Corollary 1.3}

Proof of Theorem 1.2. We proceed by several steps.

Step 1. First we prove that $g \geq \frac{d^{2}}{2 s}+\frac{d}{2 s}(2 P-2-s)-\frac{s^{3}}{2}$, where $P=P(s, t)$ is the number defined in Section 4, (ii).

Let $\Sigma \subset \mathbf{P}^{3}$ be an integral curve of degree $s$, not contained in any surface of degree $<t$, and of maximal genus $P=P(s, t)$ (compare with Section 4, (ii)). Let $X \subset \mathbf{P}^{4}$ be the cone over $\Sigma$. Since $\Sigma$ is a.C.M., and $d \gg s$, there exists an integral a.C.M. curve $Y$ on $X$, of degree $d$ [2, Lemma 18]. Since $d \gg s$, Bezout's theorem implies that $Y$ is not contained in surfaces of degree $<s$. Moreover, $Y$ is not contained in a hypersurface of degree $<t$, otherwise this hypersurface would contain $X$ because $d \gg s$, and this is not possible, for $\Sigma$ is not contained in a surface of degree $<t$. Therefore, $Y$ satisfies the flag condition of type $(s, t)$, hence $p_{a}(Y) \leq g$, for $g$ is maximal. On the other hand, since $Y$ is a.C.M., we have $p_{a}(Y)=\sum_{i=1}^{+\infty} d-h_{Y^{\prime}}(i)\left(Y^{\prime}:=\right.$ general hyperplane section of $\left.Y\right)$, and so, by [4, Lemma, $(2.1)]$, we have $p_{a}(Y) \geq \frac{d^{2}}{2 s}+\frac{d}{2 s}(2 P-2-s)-\frac{s^{3}}{2}$, hence $g \geq$ $\frac{d^{2}}{2 s}+\frac{d}{2 s}(2 P-2-s)-\frac{s^{3}}{2}$.

Step 2. Next we prove (2), and that $C$ lies on a flag $S \subset F$, where $S$ is a surface of degree s, $F$ a hypersurface of degree $t, S$ is unique, and the general hyperplane section of $S$ is a space extremal curve not contained in any surface of degree $<t$.

If $C$ were not contained in a surface of degree $s$, then, by Theorem 1.1] (and [2, Theorem] in the case $\left.s=t^{2}-t\right)$, one would have $g \leq G(d, s+1, t)+4 t^{3}$. Since $G(d, s+1, t)=\frac{d^{2}}{2(s+1)}+O(d)$, by previous step it would follow $\frac{d^{2}}{2 s}+O(d) \leq \frac{d^{2}}{2(s+1)}+$ $O(d)$. This is in contrast with the assumption $d \gg s$ (a direct and elementary computation shows that we may assume $d>s^{4}$ ). Therefore, there exists a surface $S$ of degree $s$ containing $C$. Bezout's theorem implies this surface is unique, because $d \gg s$. Let $\Sigma$ be the general hyperplane section of $S$. Since $S$ is not contained in a hypersurface of degree $<t$, and $s \geq t^{2}-2 t+3$, then, by Roth's theorem [8, (C), p. 2], $\Sigma$ is not contained in a surface of degree $<t$. Therefore, $p_{a}(\Sigma) \leq P$. On the other 
hand, since $C \subset S$, by [4, Lemma, (2.1)], we have $g \leq \frac{d^{2}}{2 s}+\frac{d}{2 s}\left(2 p_{a}(\Sigma)-2-s\right)+\frac{s^{3}}{2}$. By Step 1 we deduce:

$$
\frac{d^{2}}{2 s}+\frac{d}{2 s}(2 P-2-s)-\frac{s^{3}}{2} \leq g \leq \frac{d^{2}}{2 s}+\frac{d}{2 s}\left(2 p_{a}(\Sigma)-2-s\right)+\frac{s^{3}}{2} .
$$

Since $d>s^{4}$, and $p_{a}(\Sigma) \leq P$, it follows that $p_{a}(\Sigma)=P$. Taking into account (6) and (12), and that $p_{a}(\Sigma)=P$, from previous inequalities we deduce (2). Moreover, since $p_{a}(\Sigma)=P, \Sigma$ is an extremal curve. In particular $\Sigma$ is a.C.M., and lies on a space surface $F^{\prime}$ of degree $t$. It follows that also $S$ is a.C.M., and $F^{\prime}$ lifts to a hypersurface $F$ of degree $t$ containing $S$.

Step 3. C is a.C.M..

Let $S$ be the surface of degree $s$ containing $C$, as above. Let $\Gamma$ and $\Sigma$ be the general hyperplane sections of $C$ and $S$. Let $X \subset \mathbf{P}^{4}$ be the cone over $\Sigma$. By [2, Lemma 18], we know there exists an integral a.C.M. curve $B$ on $X$, whose general hyperplane section $B^{\prime}$ has the same Hilbert function as $\Gamma$. The curve $B$ satisfies the flag condition of type $(s, t)$, because $\operatorname{deg} B=d \gg s$, and $\Sigma$ is not contained in a surface of degree $<t$. Therefore, we have $p_{a}(B) \leq g$. It follows that

$$
p_{a}(B)=\sum_{i=1}^{+\infty} d-h_{B^{\prime}}(i)=\sum_{i=1}^{+\infty} d-h_{\Gamma}(i) \leq g .
$$

Since in general we have $g \leq \sum_{i=1}^{+\infty} d-h_{\Gamma}(i)$, we deduce $g=\sum_{i=1}^{+\infty} d-h_{\Gamma}(i)$. This proves that $C$ is a.C.M.

Step 4. The case $d=(m+1) s$.

Let $S$ be the surface of degree $s$ containing $C$, as above. Let $\Sigma$ denote the general hyperplane section of $S . \Sigma$ is an a.C.M. space curve, not contained in any surface of degree $<t$, with maximal genus $P=P(s, t)$. Let $B=S \cap F_{m+1}$ be a complete intersection of $S$ with a hypersurface of degree $m+1$, and $B^{\prime}$ its general hyperplane section. From the exact sequence $0 \rightarrow \mathcal{O}_{S}(-m-1) \rightarrow \mathcal{O}_{S} \rightarrow \mathcal{O}_{B} \rightarrow 0$, we get the following relation between the Hilbert polynomials of $S$ and $B: p_{B}(t)=$ $p_{S}(t)-p_{S}(t-m-1)$. Taking into account that $p_{S}(t)=s\left(\begin{array}{c}t+1 \\ 2\end{array}\right)+t(1-P)+1+p_{a}(S)$, we deduce (compare with (13)):

$$
p_{a}(B)=\frac{d^{2}}{2 s}+\frac{d}{2 s}(2 P-2-s)+1=G(d, s, t)-\frac{d}{s}(\beta-1) .
$$

Passing to the hyperplane sections, we also have the exact sequence $0 \rightarrow \mathcal{I}_{\Sigma, \mathbf{P}^{3}} \rightarrow$ $\mathcal{I}_{B^{\prime}, \mathbf{P}^{3}} \rightarrow \mathcal{O}_{\Sigma}(-m-1) \rightarrow 0$. Taking into account that $\Sigma$ is a.C.M., we deduce the following relation between the Hilbert functions: for every integer $n, h_{B^{\prime}}(n)=$ $h_{\Sigma}(n)-h_{\Sigma}(n-m-1)$.

Now we observe that, in order to prove the claim, it suffices to prove that

$$
h_{\Gamma}(n) \geq h_{B^{\prime}}(n)
$$

for every integer $n$, where $\Gamma$ denotes the general hyperplane section of $C$. In fact, since $B$ is a.C.M., from previous inequality (14), we deduce $g=\sum_{i=1}^{+\infty} d-h_{\Gamma}(i) \leq$ $\sum_{i=1}^{+\infty} d-h_{B^{\prime}}(i)=p_{a}(B)$. On the other hand, since $B$ satisfies the flag condition of type $(s, t)$, we also have $p_{a}(B) \leq g$. Therefore $g=p_{a}(B)=G(d, s, t)-\frac{d}{s}(\beta-1)$, 
and $h_{\Gamma}(n)=h_{B^{\prime}}(n)$ for every $n$. In particular, we have $h_{\Gamma}(m+1)=h_{B^{\prime}}(m+1)=$ $h_{\Sigma}(m+1)-h_{\Sigma}(0)=h_{\Sigma}(m+1)-1$. This implies there exists a surface of degree $m+1$ containing $\Gamma$, and not containing $\Sigma$. Since $C$ is a.C.M., this surface lifts to a hypersurface $G$ of degree $m+1$, containing $C$ and not containing $S$. Since $S$ is Cohen-Macaulay, by degree reasons it follows that $C$ is equal to the complete intersection $S \cap G$.

In order to prove (14), we argue as follows. First, we observe that, when $n \leq m$, we have $h_{\Gamma}(n)=h_{B^{\prime}}(n)$ because both are equal to $h_{\Sigma}(n)$, by degree reasons. Hence, we only have to examine the case $n \geq m+1$. Now, from the equality $h_{B^{\prime}}(n)=h_{\Sigma}(n)-h_{\Sigma}(n-m-1)$, passing to the difference, we get

$$
\Delta h_{B^{\prime}}(n)=h(n)-h(n-m-1)
$$

for every integer $n$, where the function $h=h(n)$ denotes the Hilbert function of the general plane section of $\Sigma$ (see (9)). Observe that, since $d \gg s$, we have $h(n)=s$ for $n \geq m$. Using (9), a direct computation proves that, for every $n \geq m$, one has

$$
s-h(n-m-1)=F_{a, b}(n),
$$

where $F_{a, b}(n)$ is the function defined in [2, Definition 13, p. 129], with $a=t-1$, and $b=m+t$. Therefore, we may write

$$
h_{B^{\prime}}(n)=\sum_{i=0}^{m} h(i)+\sum_{i=m+1}^{n} F_{a, b}(i)
$$

for every $n$. Then we may prove the inequality (14), i.e.

$$
h_{\Gamma}(n) \geq h_{B^{\prime}}(n)=\sum_{i=0}^{m} h(i)+\sum_{i=m+1}^{n} F_{a, b}(i),
$$

with the same argument as in the proof of [2, Proposition 15, p. 130], taking into account that 2, Proposition 8, p. 127], 2, Proposition 12, p. 129] and [2, Lemma 14, p. 130] hold true also for $s \geq t^{2}-2 t+3$. (compare with [2, Remark 10, p. 128]).

Proof of Corollary 1.3. Let $g$ be the arithmetic genus of $C$. Since $d \cdot e(C) \leq 2 g-2$, by Theorem 1.1 we deduce

$$
e(C) \leq \frac{2\left(G(d, s, t)+4 t^{3}\right)-2}{d},
$$

from which (3) follows, taking into account the definition of $G(d, s, t)$ (Section 2, (ii)), (6), and that $d \gg 0$.

If $d=(m+1) s$ and $s<t^{2}-t$, from (3) we get (4).

The bound (4) holds true also if $s=t^{2}-t$, because, in this case, instead of the bound $g \leq G(d, s, t)+4 t^{3}$ given by Theorem 1.1, we may apply the more fine bound $g \leq G(d, s, t)-\frac{d}{s}(\beta-1)$ given by Theorem 1.2 . 
In the range $t^{2}-2 t+3 \leq s \leq t^{2}-t$, the bound (4) is sharp. In fact, let $B$ be a complete intersection on a surface as in the claim, and denote by $B^{\prime}$ its general hyperplane section. Then $B$ is a.C.M., and therefore

$$
e(B)=\max \left\{n: h_{B^{\prime}}(n)<d\right\}-1 \text {. }
$$

Combining with (15) and (10), we get $e(B)=\frac{d}{s}+2 t-7$.

\section{Appendix}

We keep all the notation stated in Section 1 and 2 .

(i) The function $\rho=\rho(s, t, \epsilon)$ is defined as follows (see [2, p. 120]).

If $\epsilon \geq s-(\beta+1)(\alpha+\beta+2-t)$, divide $s-\epsilon-1=u(\alpha+\beta+2-t)+v$, and put:

$$
\begin{aligned}
\rho:= & \frac{s-1-\epsilon}{s}\left[\frac{s^{2}}{2 t}+\frac{s}{2}(t-4)-\frac{(t-1-\beta)(1+\beta)(t-1)}{2 t}+1\right]+\frac{1+\epsilon}{2 s}(s-\epsilon+1) \\
& +\left(\begin{array}{c}
u+v+1 \\
2
\end{array}\right)-\frac{1}{2}(\alpha+\beta)\left(2 v+u \alpha+u \beta-u^{2}\right)+\frac{1}{2} u(t-1)(t-\beta-3)-1 ;
\end{aligned}
$$

if $\epsilon<s-(\beta+1)(\alpha+\beta+2-t)$, divide $\epsilon=u(\alpha+\beta+1)+v$, and put:

$$
\begin{gathered}
\rho:=\frac{s-1-\epsilon}{s}\left[\frac{s^{2}}{2 t}+\frac{s}{2}(t-4)-\frac{(t-1-\beta)(1+\beta)(t-1)}{2 t}+1\right]+\frac{1+\epsilon}{2 s}(s-\epsilon+1) \\
-\frac{1}{2}(\alpha+\beta)(t-u-1)(\alpha+t+u-3)+\frac{1}{2} \beta(t-1)(2 \alpha-6)-1 .
\end{gathered}
$$

Similarly, we define $\rho^{\prime}=\rho(s, \tau, \epsilon)$ (compare with Section 2, (i)).

(ii) The number $R$ appearing in (7) is defined as follows ([1], 5, p. 91-92, (4) and $\left.\left.\left(4^{\prime}\right)\right]\right)$.

First, define $k$ and $\delta$ by dividing $\epsilon=k w+\delta, 0 \leq \delta<w$, when $\epsilon<\left(3-w_{1}\right) w$. Otherwise, define $k$ and $\delta$ by dividing $\epsilon+2-w_{1}=k(w+1)+\delta, 0 \leq \delta<w+1$. Then we have:

$$
R:=\frac{1+\epsilon}{2 s}(s+1-\epsilon-2 \pi)+w(\epsilon-\delta)-k\left(\begin{array}{c}
w+1 \\
2
\end{array}\right)+\left(\begin{array}{l}
\delta \\
2
\end{array}\right) .
$$

(iii) Sketch of the proof of (66). We only prove that $|\rho| \leq 2 t^{3}$ in the case $\epsilon \geq$ $s-(\beta+1)(\alpha+\beta+2-t)$. The analysis of the case $\epsilon<s-(\beta+1)(\alpha+\beta+2-t)$, and the proof of the estimate $\left|\rho^{\prime}\right| \leq 2 t^{3}$, are quite similar, therefore we omit them.

Set:

$$
H:=H(s, t):=\frac{s^{2}}{2 t}+\frac{s}{2}(t-4)-\frac{(t-1-\beta)(1+\beta)(t-1)}{2 t}+1 .
$$

This number is the coefficient of the term $\frac{s-1-\epsilon}{s}$ appearing in the definition of $\rho$. By the way, notice that, if $s>t^{2}-t$, then $H$ is the Halphen's bound for the genus

\footnotetext{
${ }^{1}$ In the formula defining $\rho$ in [2, p. 120], there is a misprint. In fact, in the case $\epsilon \geq s-(\beta+$ $1)(\alpha+\beta+2-t)$, the factor $\alpha-\beta-6$ must be replaced by $t-\beta-3$ (compare with [3 p. 2708], line 10 from below).
} 
of a space curve of degree $s$, not contained in any surface of degree $<t([9$, p. 1$]$, [7, 10.8. Teorema, p. 56]). We also notice we may write:

$$
G(d, s, t)=\frac{d^{2}}{2 s}+\frac{d}{2 s}(2 H-2-s)+\rho+1 .
$$

Taking into account that $s-1=\alpha t+\beta$, we may rewrite $H$ :

$$
2 H=\alpha t^{2}+\left(\alpha^{2}-4 \alpha\right) t+\beta^{2}+2 \alpha+(2 \alpha-1) \beta .
$$

The function $\alpha \rightarrow \alpha^{2}-4 \alpha$ is growing for $\alpha \geq 2$. Therefore, when $\alpha \geq 2$, since $0 \leq \alpha \leq t-2$ and $0 \leq \beta \leq t-1$, it follows that:

$$
\begin{aligned}
0 \leq 2 H \leq( & t-2) t^{2}+\left((t-2)^{2}-4(t-2)\right) t+(t-1)^{2}+ \\
& +2(t-2)+(2(t-2)-1)(t-1)=2\left(t^{3}-\frac{7}{2} t^{2}+\frac{5}{2} t+1\right) .
\end{aligned}
$$

This inequality holds true also for $\alpha \leq 1$. Hence

$$
0 \leq H \leq t^{3}-\frac{7}{2} t^{2}+\frac{5}{2} t+1 \text {. }
$$

Since $\frac{s-1-\epsilon}{s} \geq 0, H \geq 0$, and $t-\beta-3 \geq-2$, from the definition of $\rho$ we deduce:

$$
\rho \geq-\frac{1}{2}(\alpha+\beta)(2 v+u \alpha+u \beta)-u(t-1)-1 .
$$

Taking into account that

(21) $s \leq t^{2}-t, \quad \alpha \leq t-2, \quad v \leq \alpha+\beta+1-t \leq \beta-1 \leq t-2, \quad u \leq \beta \leq t-1$, substituting in a similar manner as in (19), it follows that

$$
\rho \geq-2 t^{3}+5 t^{2}-\frac{3}{2} t-\frac{7}{2} \geq-2 t^{3} .
$$

Moreover, since $\frac{s-1-\epsilon}{s} \leq 1$,

$$
\frac{1+\epsilon}{2 s}(s-\epsilon+1) \leq \frac{1}{2}(s+1) \leq \frac{1}{2}\left(t^{2}-t+1\right),
$$

and $u \leq \beta$ (which implies that $u \beta-u^{2} \geq 0$, so $\left.-\frac{1}{2}(\alpha+\beta)\left(2 v+u \alpha+u \beta-u^{2}\right) \leq 0\right)$, from (20), (21), and the definition of $\rho$, it follows that:

$$
\begin{gathered}
\rho \leq H+\frac{1}{2}\left(t^{2}-t+1\right)+\frac{1}{2}(u+v+1)(u+v)+\frac{1}{2} u t(t-1) \leq \\
\leq\left(t^{3}-\frac{7}{2} t^{2}+\frac{5}{2} t+1\right)+\frac{1}{2}\left(t^{2}-t+1\right)+\frac{1}{2}(2 t-2)(2 t-3)+\frac{1}{2} t(t-1)^{2}= \\
=\frac{3}{2} t^{3}-2 t^{2}-\frac{5}{2} t+\frac{9}{2} \leq 2 t^{3} .
\end{gathered}
$$

Combining this estimate with (22), we deduce $|\rho| \leq 2 t^{3}$, in the case $s \leq t^{2}-t$ and $\epsilon \geq s-(\beta+1)(\alpha+\beta+2-t)$.

(iv) Proof of (8). Recall that $s-1=2 w+w_{1}, 0 \leq w_{1} \leq 1$, and $\pi=w\left(w-1+w_{1}\right)$ (compare with Section 2, (iii), and with this Appendix, (ii)). Hence we have:

$$
s+1-\epsilon-2 \pi \leq s+1-2 \pi=\frac{1}{2}\left(-s^{2}+6 s+w_{1}^{2}-2 w_{1}-1\right) \leq \frac{1}{2}\left(6 s-s^{2}\right) .
$$


Therefore, if $s \geq 6$, then $s+1-\epsilon-2 \pi \leq 0$. In this case, taking into account that $w \leq(s-1) / 2$ and that $\delta \leq w$, we have:

$$
\begin{aligned}
R \leq w \epsilon+ & \frac{1}{2} \delta(\delta-1) \leq w(s-1)+\frac{1}{2} w(w-1) \leq \\
& \leq \frac{1}{2}(s-1)^{2}+\frac{1}{2} \frac{s-1}{2} \frac{s-3}{2} \leq \frac{5}{8}(s-1)^{2} \leq s^{2} .
\end{aligned}
$$

An easy direct computation shows that the inequality $R \leq s^{2}$ holds true also when $3 \leq s \leq 5$. Therefore we have:

$$
R \leq s^{2}
$$

On the other hand we have:

Hence:

$$
R \geq \frac{1+\epsilon}{2 s}(-\epsilon-2 \pi)-w \delta-\frac{1}{2} w(w+1) k .
$$

$$
\begin{gathered}
-R \leq \frac{1}{2}(s-1+2 \pi)+w \delta+\frac{1}{2} w(w+1) k= \\
=\frac{1}{2}\left(\frac{1}{2} s^{2}-s+\frac{1}{2}+w_{1}-\frac{1}{2} w_{1}^{2}\right)+w \delta+\frac{1}{2} w(w+1) k .
\end{gathered}
$$

When $\epsilon<w\left(3-w_{1}\right)$, then $\delta \leq w-1$ and $k \leq 2$. Therefore, in this case, from (24) we have:

$$
\begin{aligned}
-R & \leq \frac{1}{2}\left(\frac{1}{2} s^{2}-s+1\right)+w(w-1)+w(w+1)= \\
\frac{1}{2}\left(\frac{1}{2} s^{2}-s+1\right)+2 w^{2} & \leq \frac{1}{2}\left(\frac{1}{2} s^{2}-s+1\right)+2 \frac{(s-1)^{2}}{4}=\frac{3}{4} s^{2}-\frac{3}{2} s+1 \leq s^{2} .
\end{aligned}
$$

When $\epsilon \geq w\left(3-w_{1}\right)$, then $\delta \leq w$ and $k \leq \frac{2(s+1)}{s}$. From (24) we get:

$$
\begin{gathered}
-R \leq \frac{1}{2}\left(\frac{1}{2} s^{2}-s+1\right)+w^{2}+w(w+1) \frac{s+1}{s} \leq \\
\frac{1}{2}\left(\frac{1}{2} s^{2}-s+1\right)+\frac{(s-1)^{2}}{4}+\left(s^{2}-1\right) \frac{s+1}{4 s}=\frac{1}{4 s}\left(3 s^{3}-3 s^{2}+2 s-1\right) \leq s^{2} .
\end{gathered}
$$

Combining with (23), we get $|R| \leq s^{2}$.

(v) Proof of Lemma 2.1. Consider the coefficient of $\frac{d}{2}$ in the expression defining $G(d, s, t)$ and $G(d, s, \tau)$ (Section 2, (ii)):

We have:

$$
\begin{aligned}
A & :=\frac{s}{t}+t-5-\frac{(t-1-\beta)(1+\beta)(t-1)}{s t}, \\
A^{\prime} & :=\frac{s}{\tau}+\tau-5-\frac{\left(\tau-1-\beta^{\prime}\right)\left(1+\beta^{\prime}\right)(\tau-1)}{s \tau} .
\end{aligned}
$$

$$
G(d, s, \tau)=G(d, s, t)+\frac{d}{2}\left(A^{\prime}-A\right)+\left(\rho^{\prime}-\rho\right) .
$$

Observe that (compare with (16)):

$$
H=\frac{s}{2}(A+1)+1, \quad H^{\prime}=\frac{s}{2}\left(A^{\prime}+1\right)+1,
$$

where $H^{\prime}=H(s, \tau)$. Hence, by (18) (compare with Section 2, (i)), we have:

$$
A^{\prime}-A=\frac{2}{s}\left(H^{\prime}-H\right)=\frac{1}{s}\left[\alpha^{\prime} \tau^{2}+\left(\alpha^{\prime 2}-4 \alpha^{\prime}\right) \tau+\beta^{\prime 2}+2 \alpha^{\prime}+\left(2 \alpha^{\prime}-1\right) \beta^{\prime}\right]
$$




$$
-\frac{1}{s}\left[\left(\alpha t^{2}+\left(\alpha^{2}-4 \alpha\right) t+\beta^{2}+2 \alpha+(2 \alpha-1) \beta\right)\right] .
$$

Simplifying, we get:

$$
A^{\prime}-A=\frac{\alpha x}{s}(\alpha x+\alpha+x-2 t+3+2 \beta) .
$$

Hence, if $\alpha x=0$, then $A^{\prime}=A$. When $\alpha>0$ and $x>0$, since $-(t-\beta)<-x(\alpha+1)$, we have:

$$
\begin{gathered}
\alpha x+\alpha+x-2 t+3+2 \beta=\alpha x+\alpha+x-2(t-\beta)+3 \\
\leq \alpha x+\alpha+x-2(x(\alpha+1)+1)+3=(1-x)(\alpha+1) \leq 0 .
\end{gathered}
$$

If $x=1$, then the number

$$
\alpha x+\alpha+x-2 t+3+2 \beta=2 \alpha+4-2 t+2 \beta
$$

vanishes if and only if $\beta=t-\alpha-2$. Summing up, we get: in any case, one has $A^{\prime} \leq A$. Moreover, $A^{\prime}=A$ if and only if either $\alpha=0$ or $x=0$ or $x=1$ and $\beta=t-\alpha-2$, i.e. if and only if either $s \leq t$ or $s \geq t+1$ and $t-\alpha-2 \leq \beta<t$. In particular, when $A^{\prime}<A$, then $A-A^{\prime} \geq \frac{\alpha}{s} \geq \frac{1}{s t}$.

We deduce the following.

1) If $t+1 \leq s \leq t^{2}-t$ and $\beta<t-\alpha-2$, then $A^{\prime}<A$. Therefore, from (6) and (25), we deduce that $G(d, s, \tau)<G(d, s, t)$ for $d>8 s t^{4}$. In fact, in this case, we have $\frac{d}{2}\left(A^{\prime}-A\right)+\left(\rho^{\prime}-\rho\right)<0$, because $\frac{2\left(\rho^{\prime}-\rho\right)}{A-A^{\prime}} \leq 2 \cdot 4 t^{3} \cdot s t$.

2 ) If $s \leq t^{2}-t$ and $t-\alpha-2<\beta$, then $A=A^{\prime}$. Hence, (25) becomes $G(d, s, \tau)=$ $G(d, s, t)+\left(\rho^{\prime}-\rho\right)$. A direct computation, which we omit, shows that, in this case, if either $s-\epsilon-1<\alpha+\beta+2-t$ or $\beta(\alpha+\beta+2-t) \leq s-\epsilon-1<(\beta+1)(\alpha+\beta+2-t)$, then $\rho=\rho^{\prime}$. Hence, we have $G(d, s, \tau)=G(d, s, t)$.

3) If either $s \leq t$ or $t+1 \leq s \leq t^{2}-t$ and $t-\alpha-2 \leq \beta$, then $A=A^{\prime}$. Therefore, by (6) and (25), we get $G(d, s, \tau)=G(d, s, t)+\left(\rho^{\prime}-\rho\right) \leq G(d, s, t)+4 t^{3}$.

This concludes the proof of Lemma 2.1.

(vi) Proof of Lemma 2.2. Consider the coefficient of $\frac{d}{2}$ in the formula (7) defining $G$ :

$$
A^{\prime \prime}:=\frac{2 \pi-2}{s}-1
$$

We have:

$$
G=G(d, s, t)+\frac{d}{2}\left(A^{\prime \prime}-A\right)+(R-\rho-1) .
$$

A direct computation proves that:

$$
A^{\prime \prime}-A=\frac{1}{2 s}\left[\left(\alpha^{2}-2 \alpha\right) t^{2}+\left(2 \alpha \beta+6 \alpha-2 \alpha^{2}\right) t+\left(-4 \alpha-\beta^{2}-4 \alpha \beta+w_{1}\right)\right] .
$$

If $\alpha=0$, i.e. $s \leq t$, then $s=\beta+1$, and

$$
A^{\prime \prime}-A=\frac{1}{2 s}\left(-\beta^{2}+w_{1}\right) \text {. }
$$

If $t+1 \leq s \leq 2 t-3$, then $\alpha=1$, and we have:

$$
A^{\prime \prime}-A=\frac{1}{2 s}\left[-(t-\beta-2)^{2}+w_{1}\right] .
$$


In both cases we have $A^{\prime \prime}-A<-\frac{1}{2 s}$. Therefore, from (26) and (8), we deduce that $G<G(d, s, t)$ for $d>32 t^{4}$, because in this case $\frac{d}{2}\left(A^{\prime \prime}-A\right)+(R-\rho-1)<0$ (in fact: $\left.\frac{2(R-\rho-1)}{A-A^{\prime \prime}} \leq 4 s\left(s^{2}+2 t^{3}\right) \leq 32 t^{4}\right)$.

This concludes the proof of Lemma 2.2 .

Remark 6.1. (i) A similar argument shows that if $2 t-2 \leq s \leq 2 t$, then $A^{\prime \prime}=A$, and that if $t>2$ ed $s \geq 2 t+1$, then $A^{\prime \prime}>A$.

(ii) When $s \geq t+1$ and $t-\alpha-2 \leq \beta$, it may happen that $G(d, s, \tau)>G(d, s, t)$. For instance, if $s=t^{2}-2 t+6$ and $\epsilon=s-25$, then $\rho^{\prime}-\rho=2(t+1)$.

Acknowledgment. I would like to thank Luca Chiantini and Ciro Ciliberto for valuable discussions and suggestions, and their encouragement.

\section{REFERENCES}

[1] Chiantini, L. - Ciliberto, C. - Di Gennaro, V.: The genus of projective curves, Duke Math. J., 70 (2), 229-245 (1993).

[2] Chiantini, L. - Ciliberto, C. - Di Gennaro, V.: The genus of curves in $\mathbf{P}^{4}$ verifying certain flag conditions, Manuscripta Math. 88, 119-134. (1995).

[3] Ciliberto, C. - Di Gennaro, V.: Factoriality of certain threefolds complete intersections in $\mathbf{P}^{5}$ with ordinary double points, Comm. in Algebra, Vol. 32, n. 7, 2705-2710, 2004.

[4] Di Gennaro, V.: Hierarchical structure of the family of curves with maximal genus verifying flag conditions, Proc. Amer. Math. Soc., Volume 136, Number 3, March 2008, Pages 791-799.

[5] Di Gennaro, V. - Franco, D.: A speciality theorem for curves in $\mathbf{P}^{5}$, Geom. Dedicata (2007), 129, 89-99.

[6] Eisenbud, D. - Harris, J.: Curves in Projective Space, Sém. Math. Sup. 85, Les Presses de L’Université de Montréal, Montréal (1982).

[7] Ellia, P.: Introduzione alle curve dello spazio proiettivo, con la collaborazione di E. Mezzetti, Università degli Studi di Trieste, Dipartimento di Scienze Matematiche, Quaderni Matematici, II serie, p. 1-58, n. 273, luglio 1992.

[8] Ellinsgrud, G. - Peskine, C.: Sur les surfaces lisses de $\mathbf{P}_{4}$, Invent. Math., 95, p. 1-11, (1989).

[9] Gruson, L. - Peskine, C.: Genre des courbes dans l'espace projectif, Algebraic Geometry: Proceedings, Norway, 1977, Lecture Notes in Math., Springer-Verlag, New York, 687 (1978), 31-59.

[10] Gruson, L. - Peskine, C.: Postulation des courbes gauches, "Algebraic Geometry Open problems, Ravello 1982"Lecture Notes in Math., Springer-Verlag, New York, 997 (1983), 218-227.

[11] Sernesi, E.: Topics on families of projective schemes, Queen's Papers Pure Appl. Math., vol. 73, Queen's Univ., Kingston, Ont., 1986.

Università di Roma "Tor Vergata", Dipartimento di Matematica, Via della Ricerca Scientifica, 00133 Roma, Italy.

E-mail address: digennar@axp.mat.uniroma2.it 